\title{
ARTICLE OPEN \\ Far-UV photoluminescence microscope for impurity domain in hexagonal-boron-nitride single crystals by high-pressure, high-temperature synthesis
}

\author{
Kenji Watanabe iD $^{1,2 *}$ and Takashi Taniguchi ${ }^{1,2}$
}

Hexagonal-boron-nitride single crystals grown by high-pressure, high-temperature (HPHT) synthesis are commonly used as the insulated substrate dielectric for two-dimensional (2D) atomic-layered materials like graphene and transition metal dichalcogenides (TMDs) to improve the flatness of the 2D materials atomically without disturbing the 2D electronic characteristics. However, HPHT single crystals often contain impure regions, which can hold subtle clues in regard to the 2D atomic-layered materials for new discoveries in the physics of 2D materials. To identify the position of the impure domains and to avoid them when the $2 \mathrm{D}$ devices are prepared, a far-ultraviolet photoluminescence microscope was developed. This microscope makes it possible to visualize the impure-growth region with ease in a no-contact and non-destructive manner.

npj 2D Materials and Applications (2019)3:40

; https://doi.org/10.1038/s41699-019-0124-4

\section{INTRODUCTION}

Since the discovery of the exfoliation method to obtain graphene, ${ }^{1}$ two-dimensional (2D) atomic-layer materials have been extensively studied in terms of the $2 \mathrm{D}$ physics of quantum effects, superconductivity, photonics, and topology, and interest in 2D materials is expanding to transition metal dichalcogenides (TMDs), oxides, and other materials. ${ }^{2}$ From the early stage of research on graphene, hexagonal-boron nitride ( $\mathrm{h}-\mathrm{BN})$ has been known as the best substrate dielectric for studying 2D physics of graphene. ${ }^{3}$ A single crystal of h-BN has a van der Waals layered structure in which each layer is atomically flat with $\mathrm{sp}^{2}$ bonding between boron and nitrogen atoms, and the interlayers are weekly coupled via van der Waals interaction. ${ }^{4}$ The surface of the cleaved layer is thus almost free of dangling bonds and charge traps, which would scatter charged carriers and cause inhomogeneous fluctuations in potential.

Currently, our single crystals grown by high-pressure, hightemperature (HPHT) synthesis ${ }^{5}$ are most commonly used for devices used in research of $2 \mathrm{D}$ physics. ${ }^{3,6}$ Although the size of the crystals is limited to the order of square millimeters (in surface area), the HPHT single crystals formed by exfoliation using adhesive tape make it possible to obtain a thin-layer substrate dielectric with size of a few-hundred microns in area. Most studies employ them for demonstrating 2D devices, and a variety of outstanding properties and applications of atomically thin 2D materials have been reported. ${ }^{7}$ For all the success of the HPHT single crystals for 2D physics, there is still room for improvement of the HPHT crystals. One issue is the potential problem of an invisible impurity domain.

According to a study using secondary ion mass spectrometry (SIMS), carbon and oxygen atoms are easily incorporated into the single crystals. ${ }^{5}$ Such incorporation of impurities affects the luminous properties of $\mathrm{h}$-BN. Examples of cathodoluminescence $(\mathrm{CL})$ spectra at room temperature for pure and impure samples are shown in Fig. 1. Similar spectra for the impure sample are also obtained by a photoluminescence $(\mathrm{PL})$ method. $^{8}$ The spectrum for the pure sample is governed by the intense exciton luminescence at $215 \mathrm{~nm}$. In contrast, the impure sample, which contains a subtle amount of carbon and oxygen, ${ }^{5}$ shows additional luminescent structures in the region between 300 and $400 \mathrm{~nm}$ (the origin of which is still controversial. ${ }^{5,8-13}$ ) Moreover, owing to the relaxation process of excited carriers through excitons to the impurity bands, the intensity of the exciton luminescence for the impure sample is lower than that for the pure sample. ${ }^{14}$

In fact, such an impurity-incorporated domain is often contained in HPHT single crystals. A microphotograph (a), scanning electron microscope (SEM) image (b), and filtered $\mathrm{CL}$ images (c) and (d) for a relatively pure HPHT h-BN single crystal are shown in Fig. 2. In Fig. 2d, a hexagonal- or pentagonal-shaped domain appears near the center of the crystals, which have relatively high concentrations of impurities and show impurity $\mathrm{CL}$ bands at around $340 \mathrm{~nm}$. In Fig. 2c, the surrounding region of the domain is brighter and shows $215-\mathrm{nm}$ excitonic CL. The impurities in h-BN layers could potentially cause serious adverse effects within the adjacent 2D atomic layers in delicate phenomena. ${ }^{15}$ Thus, in consideration of the rapidly progressing research on $2 \mathrm{D}$ materials, a simple and user-friendly method for locating and identifying impurity distributions in the h-BN substrate dielectric is necessary. Hopefully, to control the device quality, the method can be incorporated into the exfoliation and stacking processes for 2D materials.

Since h-BN has a wide band gap and is highly transparent in the visible region, the impure domain, which shows luminescence in the ultraviolet (UV) region as described, is also invisible to the naked eye. It is impossible to distinguish the impure domain with a microscope (Fig. 2a). Thus, as shown in Fig. 2c, d, CL imaging is the most useful method for observing impure regions in crystals. However, $\mathrm{CL}$ imaging uses a vacuum chamber with electron irradiation, and h-BN shows insulation properties even in the impurity domain; therefore, during $\mathrm{CL}$ measurement, the sample becomes charged, and contamination (primarily residual hydrocarbon from the pumping system) collects on its surface. ${ }^{16}$ Thus, 
Energy (eV)

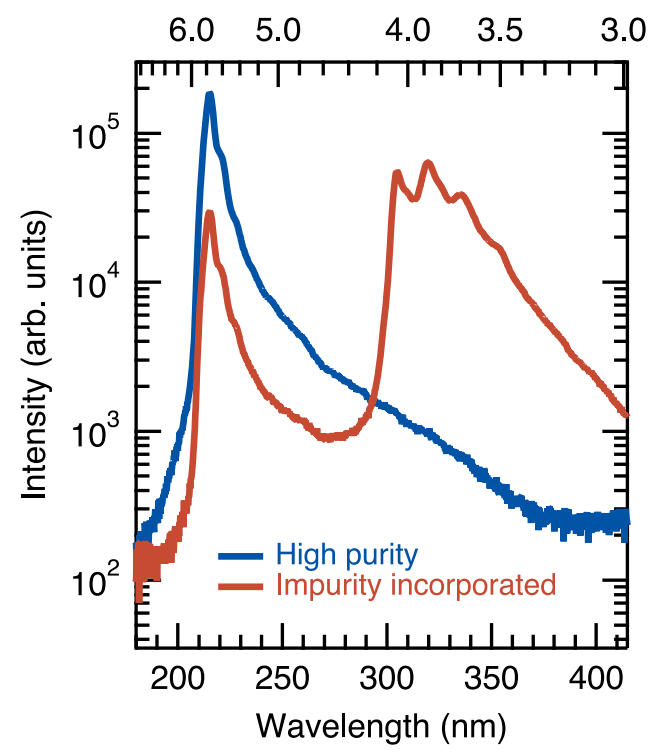

Fig. 1 Cathodoluminescence $(\mathrm{CL})$ spectra of exciton and impurity bands at room temperature. The impurity domain of the single crystal shows the impurity bands from 300 to $400 \mathrm{~nm}$ with the weak exciton band at $215 \mathrm{~nm}$

\section{(a) Microphotograph}

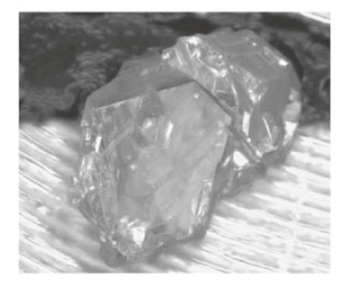

(c) $\mathrm{CL}$ (exciton)

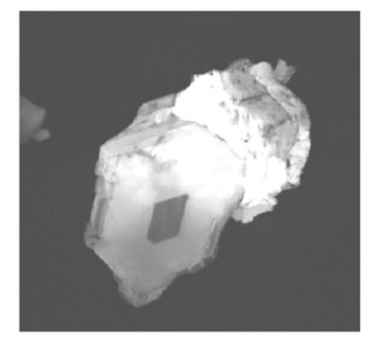

(b) SEM

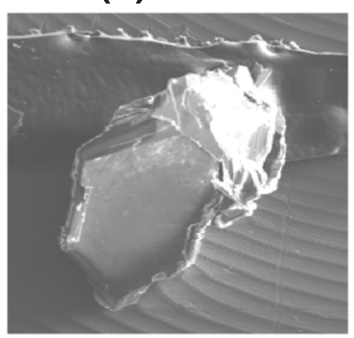

(d) CL (impurity)

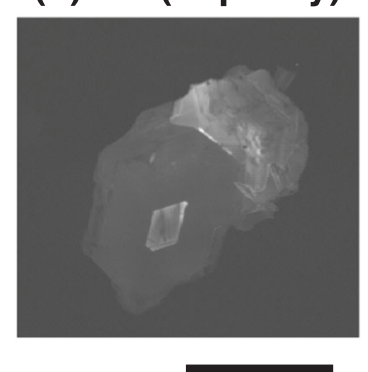

Fig. 2 Single h-BN crystal images: a microphotograph, b scanning electron microscope (SEM), c CL (exciton), and d CL (impurity). The SEM image is blurred by charging up of the insulating h-BN sample. No metal coat (to avoid electrification) was formed so that $\mathrm{CL}$ images could be observed. Images $\mathbf{b}-\mathbf{d}$ were measured by using a far-UV CL system, in which the output images have low resolution of $640 \times 480$ pixels, although the impure domain near the center of the crystal is clearly discernible in images $\mathbf{c}$ and $\mathbf{d}$. The scale bar is $500 \mu \mathrm{m}$

to use $\mathrm{CL}$ imaging to locate and identify the impure domains in preparing 2D devices requires an additional cleaning step for these contaminated surfaces prior to stacking 2D atomic layers. This contamination can be difficult to remove completely, even with wet chemical cleaning processes, which may, on the contrary, cause additional contamination.

On the other hand, a PL method using a weak far-UV excitation source can avoid the contamination problem. The impure-growth domain can be found in the small h-BN fragments resulting from exfoliation by visualizing the intensity of impurity PL band. During this visualization in a non-destructive manner, charging the sample is almost negligible in practice. In this study, we constructed a far-UV PL microscope for identifying and marking the impurity region of both bulk crystals and exfoliated thin samples on a substrate. In general, using a fluorescence microscope is an easy means to observe sample quality. However, for wide-band-gap materials such as $\mathrm{h}-\mathrm{BN}$, a unique design for the optical system is needed. This paper aims to further study 2D materials by (i) describing a simple yet powerful method for evaluating $\mathrm{h}-\mathrm{BN}$ and (ii) drawing attention to the invisible impurity-incorporated domain in HPHT single crystals.

\section{RESULTS AND DISCUSSIONS}

Far-UV PL microscope

The far-UV PL microscope is shown schematically in Fig. 3. It is composed of an excitation-light source, an objective for focusing the excitation light and collecting the luminescence from the sample, a filter box for exchanging filters, and a lens for focusing $\mathrm{PL}$ images on a charge coupled device (CCD) detector in the optical path. The sample is irradiated by the excitation light focused with the objective, and a luminescence image of the sample is collected, filtered, and then focused on the CCD detector. Designing the microscope there faced concerning dealing with the wide wavelength range of UV light (from 200 to $400 \mathrm{~nm}$ ): (1) selecting an excitation-light source at far-UV wavelength, (2) suppressing chromatic aberration in the UV range, (3) choosing optical filters usable in the UV range, and (4) detecting the weak luminescence of $\mathrm{h}-\mathrm{BN}$. The solutions to each of those challenges are described in detail in the following.

The wavelength of the excitation-light source must be shorter than that corresponding to the band-gap energy of $\mathrm{h}-\mathrm{BN}$, that is, about $6 \mathrm{eV}(206 \mathrm{~nm})$, to excite the exciton PL band. ${ }^{17,18}$ The optimal excitation wavelength must be sufficiently different from the wavelength of exciton luminescence at $215 \mathrm{~nm}$ to separate the PL component from the excitation by a far-UV interference filter. Designing the filter for a suitable transmission characteristic in this wavelength range is extremely difficult because the combinations of optical materials in the far-UV region (wavelength $<230 \mathrm{~nm}$ ) are limited. Although a synchrotron source is one of the most powerful tunable sources in the far-UV region, ${ }^{19}$ using a synchrotron radiation facility is generally limited to authorized users. It is thus unsuitable for general use such as evaluating h-BN used in the stacking process of $2 \mathrm{D}$ materials. A laser is one of the most plausible light sources. However, commercially available lasers emitting wavelengths shorter than $206 \mathrm{~nm}$ are generally expensive, and many need pulse operation with a low repetition rate, which is likely to cause damage to optical components, including mirrors, filters, and the h-BN sample. ${ }^{20}$ In view of this situation, we employed a deuterium $\left(D_{2}\right)$ lamp filtered with a double monochromator (SOL instruments Ltd., DM160) with a subtractive configuration (zero dispersion mode), and we set the filtered excitation wavelength at $198 \mathrm{~nm}$ (denoted by (1) in Fig. 3). This wavelength is the shortest UV wavelength that can propagate in the atmosphere. Using filtered light from the broader bandwidth has the advantage that excitation wavelength can be freely selected. The wavelength of $198 \mathrm{~nm}$ is relatively far from the band-gap energy of $\mathrm{h}$-BN, so it is sufficient for separating the PL component. The excitation power at the sample position was below a few $\mu \mathrm{W}$ on average. Although this lower power excitation minimized the potential for damage to the optics and samples, 


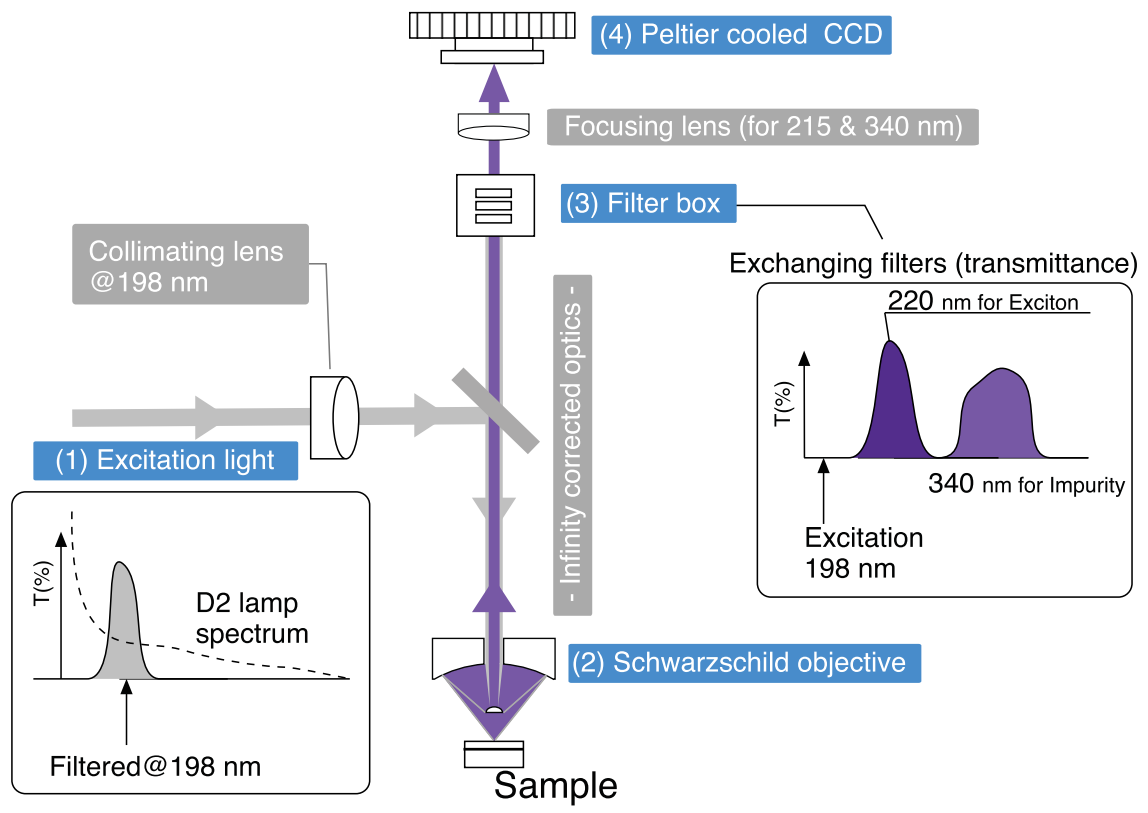

Fig. 3 Schematic diagram for far-UV photoluminescence microscope. The two insets show characteristic diagrams of the filter transmittance for the excitation source and the luminescence signal, respectively. The labels numbered from (1) to (4) denote the four technical issues explained in the text

\section{(a) $\mathrm{CL}$ (exciton)}

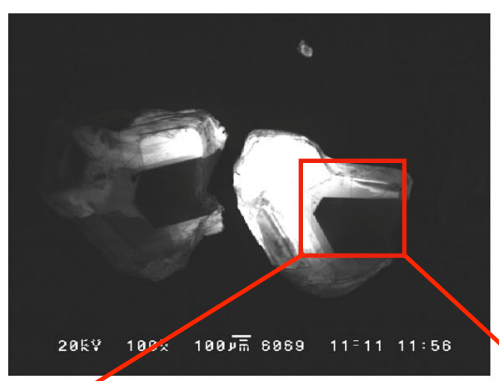

(b) CL (impurity)

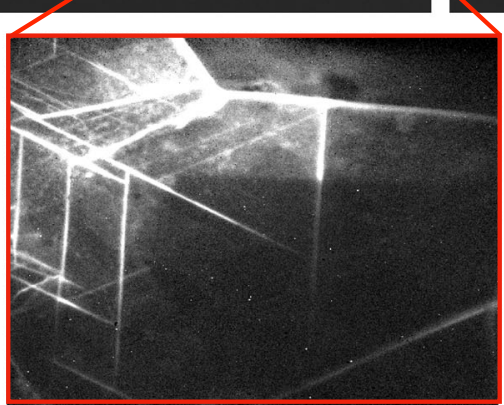

(c) PL (exciton)
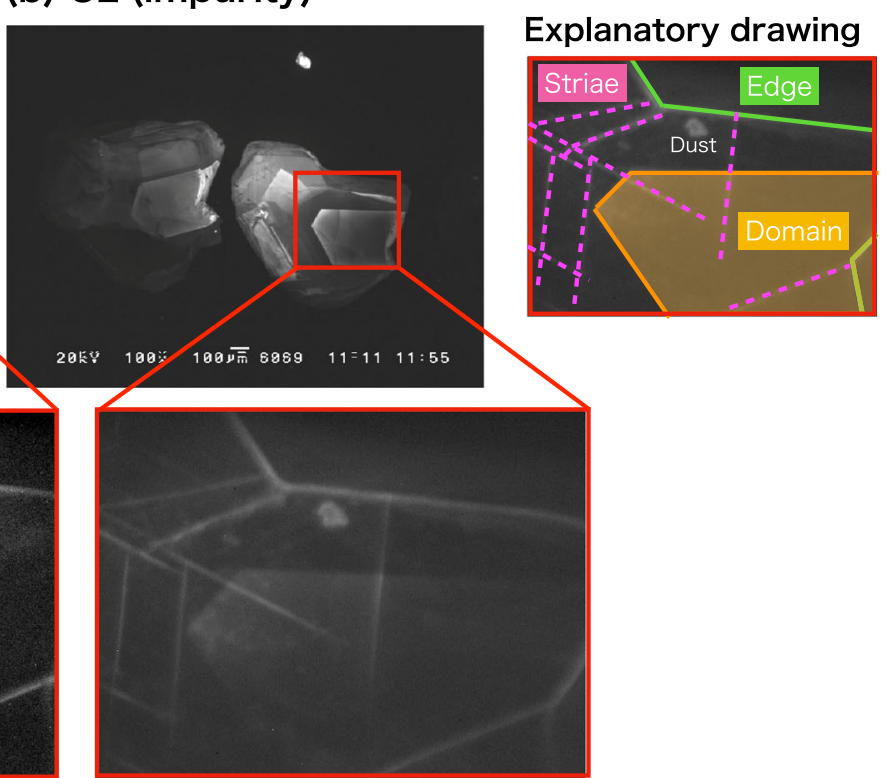

(d) PL (impurity)

Fig. 4 A comparison between $C L$ and PL images: PL images for bulk single crystal for $\mathbf{c}$ exciton band and $\mathbf{d}$ impurity band with reference $C L$ images a exciton and $\mathbf{b}$ impurity bands (an explanatory drawing is also shown). The streak lines in the PL images (denoted by pink broken lines in explanatory drawing) are scattered PL light at the striae inside the crystal. The scale bar is $200 \mu \mathrm{m}$ for $\mathbf{c}$, d

the reduced signal intensity made it difficult to obtain PL images. To detect low-PL signals, a Peltier-cooled CCD camera (Andor, Apogee Alta, denoted by (4) in Fig. 3), which can capture the subtle intensity of images with small dark-current noise over longer exposure times, was used. An exposure time of $60 \mathrm{~s}$ was sufficient to obtain the PL images, even for flakes with thickness of a few microns. Note that to gain the sensitivity in terms of luminescence, the CCD pixels were merged a block of $2 \times 2$ pixels (4 pixels) for each channel. In Figs. 4c, d and 5, each image therefore has a maximum of $512 \times 512$ channels (merged pixels). In addition, in Fig. 6, the edges of the PL images are trimmed, and the number of pixels is less than that of the other images. The PL images thus show low resolution and poor quality, although it is enough to locate and identify impurity distributions in h-BN.

An infinity-corrected optical system was incorporated in the microscope. It was thus possible to install auxiliary optical components in the parallel optical path between the objective lens and the condenser lens for CCD imaging with minimal error in 
(a) PL (exciton)

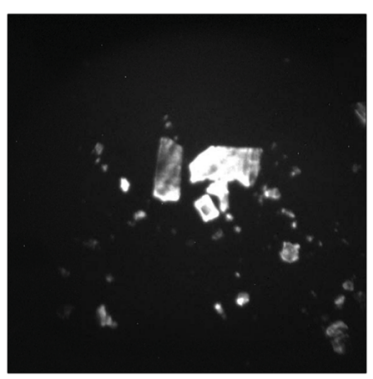

(b) PL (impurity)

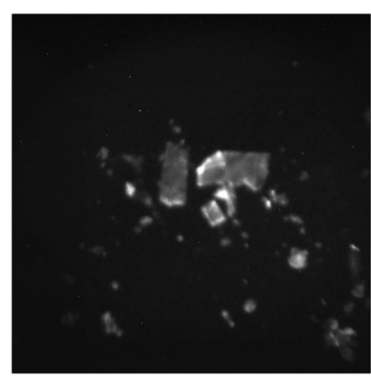

(c) Masked Image

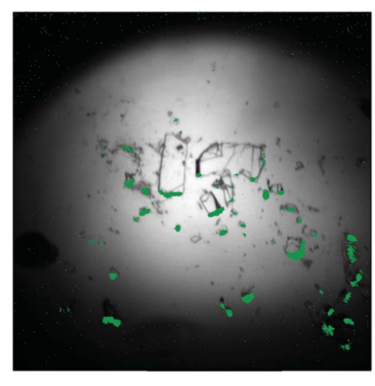

Fig. 5 PL images for exfoliated single crystal layers on Si substrate for $\mathbf{a}$ exciton band and $\mathbf{b}$ impurity band. It is difficult to judge which regions are the impurity domains. c Green masked image indicating the impurity domain overlaid on a white-light image. The scale bar is $200 \mu m$

\section{(a) Microphotograph}

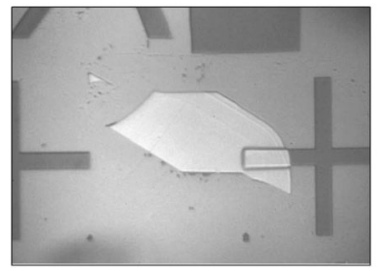

(b) Masked image

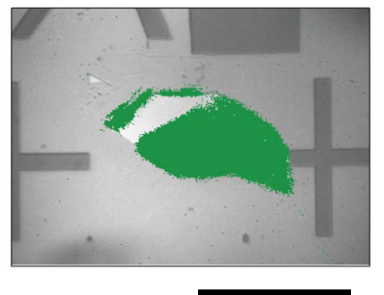

Fig. 6 Example of evaluation for exfoliated single crystal on $\mathrm{SiO}_{2}$ substrate: a microphotograph image and $\mathbf{b}$ overlay image with green mask corresponding to the area in which the impurity band is brighter than the exciton band. Dark-grey shapes are metal patterns for indicating the position of the whole substrate. The scale bar is $50 \mu \mathrm{m}$

focus. The microscope was thus highly flexible in regard to configuring optical components such as an epi-illuminator and filters.

Chromatic aberration of optics between near 200 and $400 \mathrm{~nm}$ is the most serious problem affecting imaging with the developed microscope. It is caused by the widely varying refractive indices at wavelengths from 200 to $400 \mathrm{~nm}$ of lens components for focusing. Accordingly, a Schwarzschild objective, ${ }^{21}$ which is composed of two opposing concave and convex mirrors for collimation (denoted by (2) in Fig. 3) was introduced. As a result, the objective suffers virtually no chromatic aberration. Two objectives, one for magnification of $\times 15$ and one for that of $\times 40$, were used depending on the sample size. An achromatic-lens unit (designed for wavelengths of 215 and $340 \mathrm{~nm}$ ) was used to collimate the signal image on the CCD detector. The lens unit is composed of two convex $\left(\mathrm{CaF}_{2}\right)$ lenses and one concave (quartz) lens configured for a focal length of $360 \mathrm{~mm}$. Note that it is possible to design an achromatic lens with a wavelength range for this longer focal length.

The exchanging filters selecting luminescence bands are placed in the filter box (denoted by (3) in Fig. 3). The filter for the impurity band is a commercially available U-340 filter glass (HOYA Candeo Optronics Corporation), which transmits an ideal wavelength range $(300-350 \mathrm{~nm})$ covering the impurity $\mathrm{PL}$ bands. For the exciton PL image, the combination of a 220-nm narrow bandpass filter (Acton Optics \& Coatings $220 \mathrm{~nm}$ ) and a reject filter for excitation light (Asahi Spectra, custom-made filter) was used. The 220-nm filter transmits the exciton PL signal at $\sim 215 \mathrm{~nm}$ and blocks the impurity PL signal at $\sim 340 \mathrm{~nm}$. The Asahi Spectra's filter blocks the 198-nm excitation light and transmits the exciton PL band.
Measuring bulk samples

Example PL images of the HPHT single crystal are shown in Fig. 4. As shown in Fig. 4c, similar to the exciton CL image (Fig. 4a), the PL image of the exciton band is darker at the center of the crystal indicating the impure domain; conversely, the $\mathrm{PL}$ and $\mathrm{CL}$ images of the impurity band (Fig. 4b, d) show brighter at the domain. The images obtained by $\mathrm{CL}$ and PL imaging are essentially the same. More specifically, striae in the crystal appeared as bright lines in the PL image, which can be attributed to the scatter of the PL components propagating inside the crystal. Since the $\mathrm{CL}$ images are synchronously obtained by scanning the excitation electron beam, such scattering is weaker than that due to direct excitation by the electron beam, and it is considered negligible. Employing this PL property makes it possible to visualize structural defects of excitation-wavelength size and characterize them by this PL imaging method. For exfoliation to obtain a larger single-domain flake, PL could also be used to select a bulk crystal with fewer striae.

\section{Measuring exfoliated samples}

Example PL images for exfoliated h-BN on a Si substrate are shown in Fig. 5. Unlike the PL images of bulk samples in Fig. 4, the intensity of these PL images is distributed throughout the flakes, and it is difficult to distinguish the proportion of flakes showing intense exciton or impurity PL bands. This distribution of the intensity is attributed to the exfoliated flakes have a variety of thicknesses and crystallinity characteristics, caused by differing positions of single crystals or by entirely different crystals, resulting in each flake showing a variety of intensities of the exciton and impurity bands.

To overcome the above-described difficulty, the intensity ratios of exciton images and impurity images were compared, and a mask image, in which the intensity of the impurity image was larger than that of the exciton image by a threshold value was formed. The threshold value was determined by using the intensity ratio obtained from the bulk crystals, as shown in Fig. 4c, d, in such a way that the mask image properly matched the impure region. As a result, computer-aided overlay of the mask image yielded the position of the impurity domain. The result of comparing Fig. 5a, b overlaid on a white-light image is shown in Fig. 5c. In this case the impure regions denoted by green masks are scattered over the observation area. As a more specific example, the exfoliated h-BN on a $\mathrm{SiO}_{2}$ substrate is shown in Fig. 6. At a glance, the flake at the center of the microphotograph in Fig. 6a appears to have a uniform surface; however the PL microscope judged that over $80 \%$ of the region is covered by the impurity domain, as shown in Fig. 6b, where the green area indicates the impurity PL intensity is relatively greater than that of the exciton. Thus, the flake shown in Fig. 6 is likely to have originated from the bulk sample near the center impurity domain, as shown in Fig. 4. 
In summary the use of the developed far-UV fluorescence microscope provides an easy means to observe crystal sample quality of wide-band-gap materials such as h-BN. This microscope is in fact a powerful tool for evaluating the stacking process of $2 \mathrm{D}$ materials by distinguishing impurity-incorporated regions without contact in a non-destructive manner. It also makes it possible to obtain almost-ideal interfaces for h-BN and 2D atomic layers. In addition, incorporating this microscope evaluation into the autonomous robotic identification and assembly of 2D crystals ${ }^{22}$ would greatly enhance processing high-quality van der Waals superlattices.

This simple microscope using PL images is also promising for other 2D materials, such as TMDs, as well as other wide-band-gap materials, such as AIGaN-based materials, in addition to h-BN because of the widely tunable excitation-light source and small chromatic aberration of the microscope.

\section{METHODS}

Sample preparation

h-BN single crystals were synthesized by HPHT method at $4.5 \mathrm{GPa}$ and $1500^{\circ} \mathrm{C}$ using barium boron nitride as a solvent. SIMS was employed to estimate concentration of carbon and oxygen impurities. Details are described in ref. 5.

h-BN flakes were mechanically cleaved and exfoliated on cleaned $\mathrm{Si}$ substrate (the sample represented in Fig. 5) and $\mathrm{SiO}_{2} / \mathrm{Si}$ substrate (the sample represented in Fig. 6). Both substrates did not show remarkable luminescence in the UV region which affected the PL imaging of h-BN samples.

\section{DATA AVAILABILITY}

The data supporting the findings of this work are available from the corresponding author upon reasonable request.

\section{CODE AVAILABILITY}

The code used to analyze the data are available from the corresponding author upon reasonable request.

Received: 4 July 2019; Accepted: 4 October 2019;

Published online: 25 October 2019

\section{REFERENCES}

1. Novoselov, K. S. et al. Electric field effect in atomically thin carbon films. Science 306, 666-669 (2004).

2. Geim, A. K. \& Grigorieva, I. V. Van der Waals heterostructures. Nature 499, 419-425 (2013)

3. Dean, C. R. et al. Boron nitride substrates for high-quality graphene electronics. Nat. Nanotechnol. 5, 722-726 (2010).

4. Mishima, O. \& Era, K. In Electric Refractory Materials (ed. Kumashiro, Y.) 495-556 (Marcel Dekker, Inc., New York, Basel, 2000).

5. Taniguchi, T. \& Watanabe, K. Synthesis of high-purity boron nitride single crystals under high pressure by using Ba-BN Solvent. J. Cryst. Growth 303, 525-529 (2007).

6. Wang, L. et al. One-dimensional electrical contact to a two-dimensional material. Science 342, 614-617 (2013).

7. Yankowitz, M., Ma, Q., Jarillo-Herrero, P. \& LeRoy, B. J. van der Waals heterostructures combining graphene and hexagonal boron nitride. Nat. Rev. Phys. 1 112-125 (2019).

8. Era, K., Minami, F. \& Kuzuba, T. Fast luminescence from carbon-related defects of hexagonal boron nitride. J. Lumin. 24-25, 71-74 (1981).

9. Museur, L., Feldbach, E. \& Kanaev, A. Defect-related photoluminescence of hexagonal boron nitride. Phys. Rev. B 78, 155204 (2008).

10. Du, X. Z., Li, J., Lin, J. Y. \& Jiang, H. X. The origin of deep-level impurity transitions in hexagonal boron nitride. Appl. Phys. Lett. 106, 021110 (2015).
11. Weston, L., Wickramaratne, D., Mackoit, M., Alkauskas, A. \& Van de Walle, C. G. Native point defects and impurities in hexagonal boron nitride. Phys. Rev. B 97 214104 (2018).

12. Grenadier, S. J., Maity, A., Li, J., Lin, J. Y. \& Jiang, H. X. Origin and roles of oxygen impurities in hexagonal boron nitride epilayers. Appl. Phys. Lett. 112, 162103 (2018).

13. Vokhmintsev, A., Weinstein, I. \& Zamyatin, D. Electron-phonon interactions in subband excited photoluminescence of hexagonal boron nitride. J. Lumin. 208 363-370 (2019).

14. Watanabe, K. \& Taniguchi, T. Hexagonal boron nitride as a new ultraviolet luminescent material and its application. Int. J. Appl. Ceram. Technol. 8, 977-989 (2011).

15. Wong, D. et al. Characterization and manipulation of individual defects in insulating hexagonal boron nitride using scanning tunnelling microscopy. Nat. Nanotechol. 10, 949-953 (2015).

16. Wanzenboeck, H. D., Roediger, P., Hochleitner, G., Bertagnolli, E. \& Buehler, W. Novel method for cleaning a vacuum chamber from hydrocarbon contamination. J. Vac. Sci. Technol. A 28, 1413-1420 (2010).

17. Cassabois, G., Valvin, P. \& Gil, B. Hexagonal boron nitride is an indirect bandgap semiconductor. Nat. Photonics 10, 262-266 (2016).

18. Schué, L. et al. Bright luminescence from indirect and strongly bound excitons in h-BN. Phys. Rev. Lett. 122, 067401 (2019).

19. Hua, Li, L. et al. Photoluminescence of boron nitride nanosheets exfoliated by ball milling. Appl. Phys. Lett. 100, 261108 (2012).

20. Watanabe, K. \& Taniguchi, T. Jahn-Teller effect on exciton states in hexagonal boron nitride single crystal. Phys. Rev. B 79, 193104 (2009).

21. Riedl, M. J. Optical Design Fundamentals for Infrared Systems 2nd edn (SPIE Press, Bellingham, WA, 2001).

22. Masubuchi, S. et al. Autonomous robotic searching and assembly of twodimensional crystals to build van der Waals superlattices. Nat. Commun. 9, 1413 (2018).

\section{ACKNOWLEDGEMENTS}

We would like to thank Prof. T. Machida and Ms. M. Onodera for granting us permission to use their sample photos. We also thank Mr. K. Kainuma, Atago Giken for his help in constructing the system. This research was partially supported by JSPS KAKENHI Grant Number 26286025, JP25107004, JP17H02748, the Elemental Strategy Initiative (MEXT, JAPAN), and CREST (JST, JPMJCR15F3),

\section{COMPETING INTERESTS}

The authors declare no competing interests.

\section{ADDITIONAL INFORMATION}

Correspondence and requests for materials should be addressed to K.W.

Reprints and permission information is available at http://www.nature.com/ reprints

Publisher's note Springer Nature remains neutral with regard to jurisdictional claims in published maps and institutional affiliations.

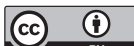

Open Access This article is licensed under a Creative Commons Attribution 4.0 International License, which permits use, sharing, adaptation, distribution and reproduction in any medium or format, as long as you give appropriate credit to the original author(s) and the source, provide a link to the Creative Commons license, and indicate if changes were made. The images or other third party material in this article are included in the article's Creative Commons license, unless indicated otherwise in a credit line to the material. If material is not included in the article's Creative Commons license and your intended use is not permitted by statutory regulation or exceeds the permitted use, you will need to obtain permission directly from the copyright holder. To view a copy of this license, visit http://creativecommons. org/licenses/by/4.0/.
}

(c) The Author(s) 2019 